\title{
Colorectal carcinoma with double somatic mismatch repair gene inactivation: clinical and pathological characteristics and response to immune checkpoint blockade
}

\author{
Tao Wang $^{1} \cdot$ Lik Hang Lee $^{1} \cdot$ Monika Vyas $^{1} \cdot$ Liying Zhang $^{1} \cdot$ Karuna Ganesh $^{2} \cdot$ Canan Firat $^{1} \cdot$ Neil H. Segal ${ }^{2} \cdot$ \\ Avni Desai ${ }^{2}$ Jaclyn F. Hechtman ${ }^{1} \cdot$ Peter Ntiamoah ${ }^{1} \cdot$ Martin R. Weiser ${ }^{3} \cdot$ Arnold J. Markowitz $^{2} \cdot$ Efsevia Vakiani $^{1}$ • \\ David S. Klimstra $^{1} \cdot$ Zsofia K. Stadler $^{2} \cdot$ Jinru Shia $\mathbb{D}^{1}$
}

Received: 18 December 2018 / Revised: 27 May 2019 / Accepted: 28 May 2019 / Published online: 7 June 2019

(c) United States \& Canadian Academy of Pathology 2019

\begin{abstract}
Double somatic mismatch-repair-gene mutation/alteration is a recently recognized molecular mechanism that underlies microsatellite instability-high in some colorectal carcinomas. It remains to be determined whether and how microsatellite instability-high tumors with this molecular defect differ from their counterparts caused by other mechanisms, specifically, Lynch syndrome-associated and MLH1-promoter hypermethylated. In this study, we evaluated the clinical and pathological characteristics of a series of 15 double somatic mutation/alteration-associated microsatellite instability-high colorectal carcinomas identified from our genetics service and 68 such cases reported in the literature. We observed that these cases presented at an age similar to MLH1-promoter hypermethylated $(n=20)$ and microsatellite-stable $(n=39)$ cases but older than Lynch syndrome-associated cases $(n=20, p<0.05)$. While these tumors simulated other microsatellite instability-high tumors in their prevalent right-sided location, they appeared to differ in TNM stages at presentation (73\% stage III/IV versus $25 \%$ stage III/IV in other microsatellite instability-high tumors, $p=0.04$ ). Histologically, $40 \%$ of them had a dominant solid growth pattern. Inter-tumoral heterogeneity was a striking feature, spanning the spectrum from medullary type (with a tumor-infiltrating-lymphocyte/high-power-field count as high as 59) to conventional-type with only few tumor-infiltratinglymphocytes (1/high-power-filed). As a group, these tumors seemed less likely to show robustly high lymphocytic infiltration than other microsatellite instability-high tumors (only $20 \%$ had $\geq 10$ tumor-infiltrating-lymphocytes/high-powerfiled, whereas this rate in Lynch syndrome-associated and $M L H 1$-promoter hypermethylated tumors was $60 \%$ and $75 \%$, respectively). Three double somatic mutation/alteration-associated tumors were treated with a PD1/PD-L1 checkpoint inhibitor. While all three had an elevated tumor-mutation-burden ( $>47 \mathrm{mut} / \mathrm{megabase})$, only one had tumor-infiltratinglymphocytes $>10 /$ high-power-field, yet all three exhibited measurable response. In summary, microsatellite instability-high colorectal carcinomas caused by double somatic mismatch-repair-gene mutation/alteration may have varied clinical and pathological characteristics, and some may have relatively low tumor-infiltrating-lymphocytes; response to immune checkpoint inhibitors can be achieved in this group even when the lymphocytic infiltration is not abundant.
\end{abstract}

$\triangle$ Zsofia K. Stadler

Stadlerz@mskcc.org

$\triangle$ Jinru Shia

Shiaj@mskcc.org

1 Department of Pathology, Memorial Sloan Kettering Cancer Center, New York, NY, USA

2 Department of Medicine, Memorial Sloan Kettering Cancer Center, New York, NY, USA

3 Department of Surgery, Memorial Sloan Kettering Cancer Center, New York, NY, USA

\section{Introduction}

Approximately $15 \%$ of colorectal carcinomas are microsatellite instability-high [1,2], a molecular phenotype that arises secondary to inactivation of one of the following DNA mismatch repair genes: MLH1, MSH2, MSH6, and $P M S 2$. The mechanism of inactivation can be hereditary or sporadic. The hereditary form is known as Lynch syndrome; these individuals have a deleterious germline mutation in the mismatch repair genes, and are at increased risk for a wide variety of cancers including colorectal, endometrial, urothelial, and other types [2,3]. Sporadic inactivation 
can occur via different mechanisms, most commonly hypermethylation of the $M L H 1$ promoter.

In recent years, the increasing use of tumor genomic sequencing in both research and clinical practice has highlighted a previously underappreciated and primarily sporadic phenomenon: double somatic mismatch repair gene inactivation resulting in microsatellite instability-high [4-6]. Unlike $M L H 1$ promoter hypermethylation which occurs by epigenetic means, double somatic inactivation results from sequence alterations (double mutations or mutation plus loss-of-heterozygosity, henceforth termed double somatic mutation/alteration). This mechanism is now recognized as the etiology for the majority of the previously "unexplained" microsatellite instability-high colorectal carcinomas, i.e., microsatellite instability-high but with no associated mismatch repair gene germline mutation or $M L H 1$ hypermethylation.

Given that the genetic background in this new group of mismatch repair-deficient tumors differs from that in tumors that have either a causative germline mutation or $\mathrm{MLH1}$ promoter methylation, it is plausible to speculate that they have different clinicopathologic traits as well. As yet, however, studies addressing this issue are scarce. Questions remain whether differences indeed exist and what the implications might be. Furthermore, while response to the recently emerged anti-PD1/PD-L1 therapeutics is expected in these double somatic mutation/alteration-associated tumors given the inherent mismatch repair deficiency, well documented examples are still lacking. In this study, we performed a comparative analysis of the clinical and pathological characteristics of this new group of microsatellite instability-high colorectal carcinomas versus other types of microsatellite instability-high tumors as well as microsatellite stable tumors. We also report on their response to anti-PD1/PD-L1 treatment.

\section{Materials and methods}

This study was approved by our Institutional Review Board. Study cases were identified from our institutional genetics databases. Selection criteria included: (1) the presence of two or more somatic mismatch repair gene mutations deemed to be at least "likely pathogenic" (see description below) or one or more such somatic mutations plus loss-ofheterozygosity, (2) mismatch repair gene deficiency as demonstrated by abnormal immunohistochemistry or microsatellite instability-high by a sequencing-based program (MSIsensor), (3) $\mathrm{MLH1}$ promoter hypermethylation negative in cases that were MLH1/PMS2-deficient, and (4) germline mismatch repair gene mutation negative. Control cases included: Lynch syndrome-associated colorectal carcinomas, defined by positive pathogenic germline mismatch repair gene mutation; $M L H 1$-methylated sporadic microsatellite instability-high colorectal carcinomas; and mismatch repair gene proficient colorectal carcinomas. All study and control cases were colorectal carcinomas either with no neoadjuvant therapy or with neoadjuvant therapy and residual viable carcinoma. All cases had material available for pathologic review.

Clinical information including anti-PD1/PD-L1 treatment was retrieved from the medical record and treating physicians' notes. In light of a recent report documenting somatic mismatch repair gene mutation in colorectal carcinomas after Hodgkin's lymphoma treatment [7], attention was also paid to whether patients had history of Hodgkin's lymphoma with treatment of infradiaphragmatic radiotherapy or procarbazine-containing chemotherapy.

Tumor hematoxylin and eosin slides were reviewed histologically and typed as conventional, mucinous, signet ring cell, or medullary. Tumor differentiation was recorded as well to moderately differentiated $(>50 \%$ with gland formation) or poorly differentiated ( $>50 \%$ lacking gland formation). Signet ring cell type or medullary type carcinomas were defined as having at least $50 \%$ of the tumor being signet ring cells or medullary. Tumor-infiltrating-lymphocytes/high-power-field was assessed over five contiguous high-power fields in the region with the highest lymphocyte density in each tumor; the mean value was taken as the representative tumor-infiltrating-lymphocyte count/highpower-field for each tumor. Peritumoral lymphoid aggregates were assessed by counting the number of discrete peri-tumoral lymphoid aggregates in a 20x field in the area with the densest number of aggregates. To explore the variations of lymphocytic infiltration, we evaluated the differences of tumor-infiltrating-lymphocytes and peritumoral lymphoid aggregates between the groups by using a low cutoff value (3 tumor-infiltrating-lymphocytes/highpower-field, 3 peritumoral lymphoid aggregates) and an arbitrarily defined high cutoff value (10 tumor-infiltratinglymphocytes/high-power-field, 10 peritumoral lymphoid aggregates) respectively.

Tumor somatic mutations were evaluated by Memorial Sloan Kettering-IMPACT, a hybridization capture based next-generation sequencing assay that assesses the coding regions of multiple cancer genes, along with selected promoters, introns, copy number status, and microsatellite status [8-10]. For the cases included in this study, the 341 and 410 cancer gene panels were utilized. Tumor mutation burden was calculated using the total number of nonsynonymous mutations divided by the total genomic target region for which mutations were reported. To determine the pathogenicity of the mismatch repair gene mutations identified on tumor sequencing, the mutations were explored using the NCBI ClinVar and OncoKB for classification of pathogenicity. Mutations that resulted in truncation or 
frameshifts were considered at least "likely pathogenic" [11]. Small indels (insertion or deletion of bases) and single nucleotide variants were inputted into in silico protein variant effect prediction algorithms, including PolyPhen-2, PROVEAN, and SIFT [11-14]. If most algorithms determined the variant to be damaging or likely damaging, then it was considered a pathogenic mutation. Mutations on POLD1 and POLE were similarly analyzed. To determine loss-of-heterozygosity events, the FACETS (Fraction and Allele-Specific Copy Number Estimates from Tumor Sequencing) program, which is an allele-specific copy number analysis pipeline and open-source software for next generation sequencing data [9], was used.

MLH1 promoter methylation was evaluated by pyrosequencing. Briefly, both tumor and normal DNA were extracted and bisulfite-treated using EZ-DNA Methylation $\mathrm{Kit}^{\mathrm{TM}}$ (Cat\#D5020, Zymo Research). A single PCR fragment spanning the target region is amplified and the degree of methylation of five $\mathrm{CpG}$ sites is analyzed in a single Pyrosequencing reaction (Qiagen). The PCR products (each $10 \mu \mathrm{l})$ were sequenced by pyrosequencing on a PyroMark Q24 Workstation (Qiagen) following the manufacturer's instructions. The $M L H 1$ hypermethylation levels were graded as positive if five of five $\mathrm{CpG}$ sites are methylated at $>=10 \%$. To confirm the somatic nature of the mismatch repair gene alterations detected in the tumor, matched germline DNA from prospectively collected blood samples was analyzed. Additionally, in cases without confirmed MLH1 promoter methylation, multigene panel germline mutation testing was also performed separately as part of the clinical diagnostic work-up and according to standard methodologies.

Colorectal carcinomas that had microsatellite instabilityhigh caused by double somatic mismatch repair gene mutation/alteration reported in the literature were identified via a search in PubMed using the parameters of ("somatic") AND ("Lynch" OR "mismatch repair" OR "microsatellite instability") AND ("colon" OR "colorectal") for studies published between 1992 and May 2018. Results were then manually reviewed for papers with adequate clinical or pathological data. To be considered comparable for our study purposes, the tumor must have double somatic mismatch repair gene inactivation through deleterious mutation (s) and/or copy number loss. Furthermore, the patient must have negative germline testing for mismatch repair gene mutation and the tumor must be negative for $M L H 1$ hypermethylation. As some studies included overlapping patient datasets, only data points that we could ensure were not duplicates were included.

Statistical analysis between groups was compared by ANOVA with Dunnett's multiple comparison adjustment for continuous variables or by Kruskal-Wallis test with Dunn's multiple comparison adjustment for non-parametric variables. Binomial variables were compared with Fisher's Exact Test with Bonferroni multiple comparison adjustment. For clinical features including age and tumor location, data from double somatic mutation/alteration cases gathered through literature review were combined with our own cohort for statistical analysis. Statistics were performed using GraphPad Prism software.

\section{Results}

\section{Study cases}

From our institutional databases, we identified 15 primary colorectal carcinomas with mismatch repair deficiency caused by double somatic mismatch repair gene mutation/alteration (henceforth referred to as "institutional cases"). As controls, we identified 79 colorectal carcinomas: 20 Lynch syndromeassociated microsatellite instability-high, $20 \mathrm{MLHI}$ promoter hypermethylated microsatellite instability-high, and 39 mismatch repair proficient (microsatellite stable). No cases had a history of Hodgkin's lymphoma (one Lynch Syndrome patient received dacarbazine along with adriamycin for a high grade retroperitoneal sarcoma 16 years prior to the development of colorectal carcinoma; one patient from the double somatic mutation/alteration group developed Burkitt lymphoma 3 years after the diagnosis of colorectal carcinoma). Our study cases and control cases allowed detailed histological assessment. From the literature, we detected 68 double somatic mutation/ alteration associated microsatellite instability-high colorectal carcinoma cases [15-19] after excluding repeated reports; these cases enriched data on patients' clinical characteristics and the affected mismatch repair genes.

\section{Characterization of institutional cases}

Molecular and genetic data including tumor-mutation burden and the pertinent somatic mutations identified in the mismatch repair genes in all 15 double somatic mutation/ alteration cases are outlined in Table 1.

The tumor mutation burden of the double somatic mutation/alteration cases ranged from 33.5 to $330.6 \mathrm{mut} /$ megabase (median, 64.9, mean, 80.4). The double somatic mutations/alterations involved different mismatch repair genes: 8 involved $\mathrm{MSH} 2$ (including one with coinactivation of $M L H 1$ by promoter methylation, a case previously reported [20]), 5 involved $M L H 1$, and 2 involved MSH6. Of the 15 cases, 5 had mismatch repair inactivation via mutation plus loss of heterozygosity; the remaining 10 had double somatic mutations. All cases were microsatellite instability-high by next generation sequencing-based MSIsensor score, and showed loss of the affected mismatch repair protein by immunohistochemistry. The somatic 


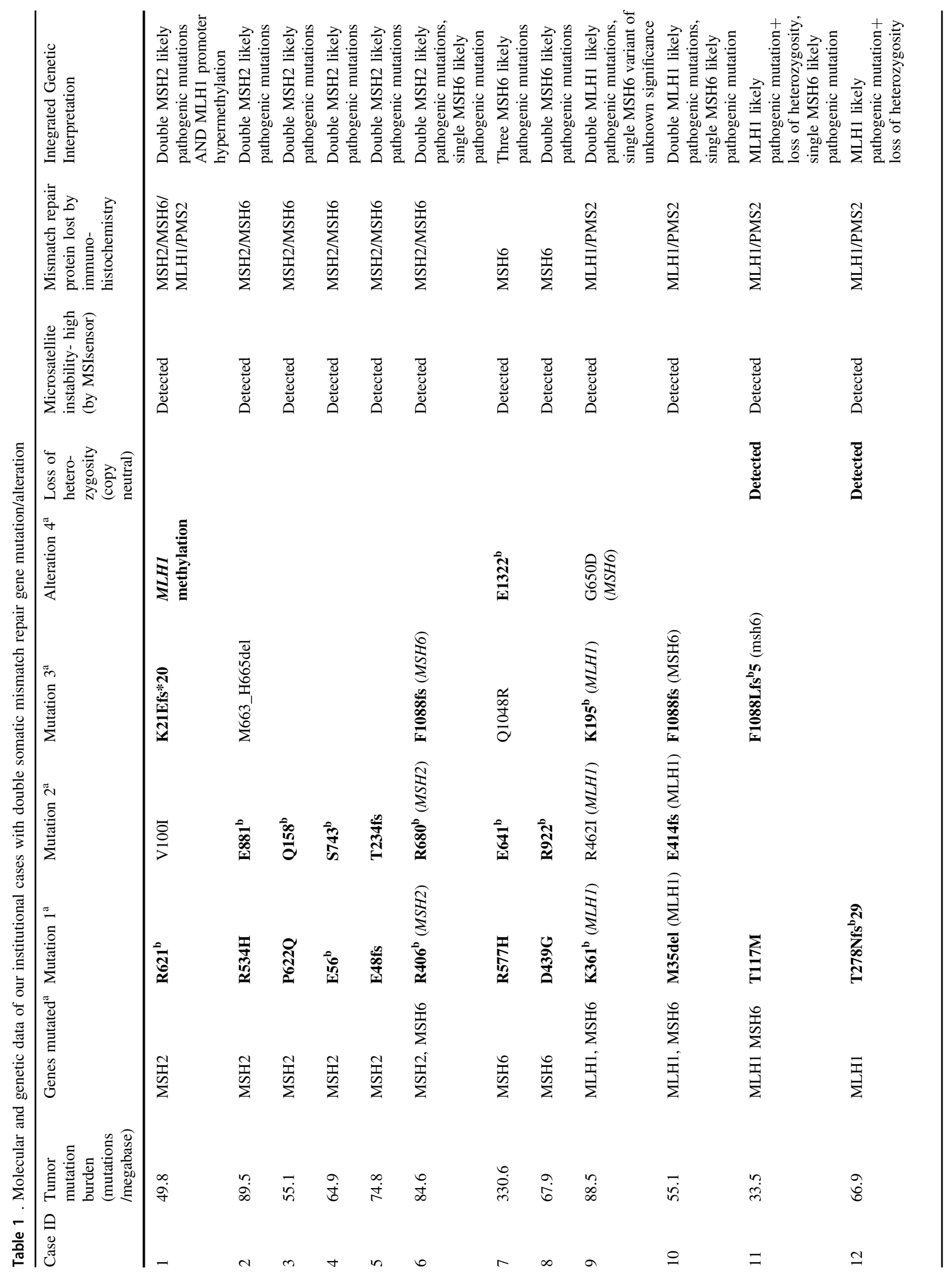




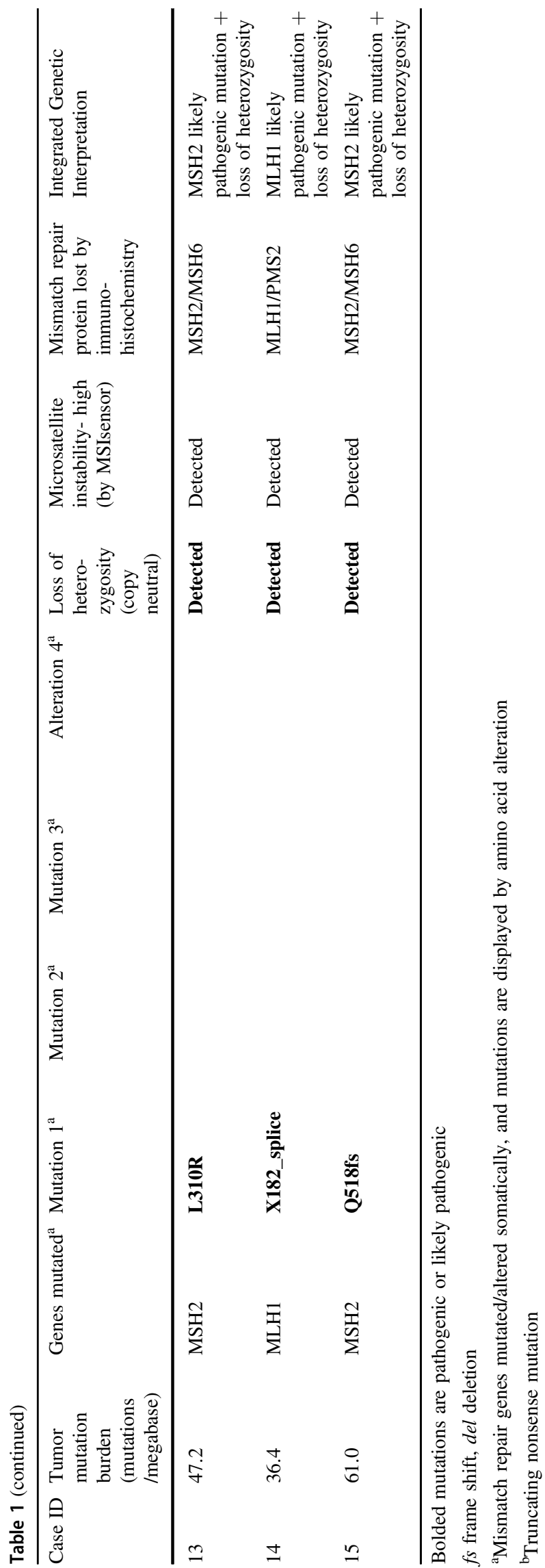

mutations in all tumors were deemed to be at least likely pathogenic, and the causative genes driving the mismatch repair deficiency in each case were determined. Three double somatic mutation/alteration cases (\#6, 10, 11), one involving $M S H 2$ and two involving $M L H 1$, also had a likely pathogenic mutation in MSH6. The specific cancer cell fraction carrying the MSH6 mutation was not calculated in this analysis.

None of the cases had germline or somatic POLDI or POLE hotspot mutations. It was noted that one of the two MSH6 inactivated cases (case 7) harbored multiple somatic non-hotspot mutations in POLE (p.N363D, p.P68H, p.F768S, p.R1471C), and this tumor had the highest tumor mutation burden of the group at 330.6/ megabase. Given that the POLE mutations are not in the known hotspots, and there was also no detectable germline defect, it was unclear whether and how any of these POLE mutations contributed to the heavy mutation burden.

It is also noteworthy that case \#1 and \#8 each had a BRAF V600E mutation in the tumor; case \#1 had MLH1promoter methylation co-existing with double somatic mutation on $M S H 2$, case \#8 had double somatic mutation on MSH6.

The clinical and pathological characteristics of the double somatic mutation/alteration cases are summarized in Table 2, and their comparison with control cases is outlined in Table 3. The double somatic mutation/alteration colorectal carcinoma cases presented at an older age than Lynch syndrome-associated cases. The male to female ratio was 2:1. Like other types of microsatellite instability-high colorectal carcinomas, double somatic mutation/alteration carcinomas exhibited a right-side dominance over left-side in tumor location $(p<0.04)$. Different from other tumors, a higher proportion of double somatic mutation/alteration colorectal carcinomas presented at an advanced TNM stage (73\% stage III/IV versus $25 \%$ each in Lynch syndrome group and MLH1 methylated group; $p=0.04$ ).

Histologically, as shown in Table 2 and illustrated in Fig. 1, double somatic mutation/alteration colorectal carcinomas had a striking tendency towards solid growth: six of the 15 tumors had $>50 \%$ of the tumor lacking gland formation; four were poorly differentiated with very low tumor-infiltrating-lymphocyte count (1-3/high-power-field) and one was medullary with 51 tumor-infiltrating-lymphocytes/high-power-field. The rest were either mucinous (20\%), or moderately differentiated gland-forming adenocarcinomas with varied lymphocyte counts. When the tumor-infiltrating-lymphocyte/high-power-field cutoff was set at 3, the proportions of double somatic mutation/ alteration tumors with high lymphocytes seemed similar to other microsatellite instability-high tumors. When the cutoff was set at 10 , only $20 \%$ double somatic mutation/alteration tumors had a count above this cutoff, whereas this rate in 


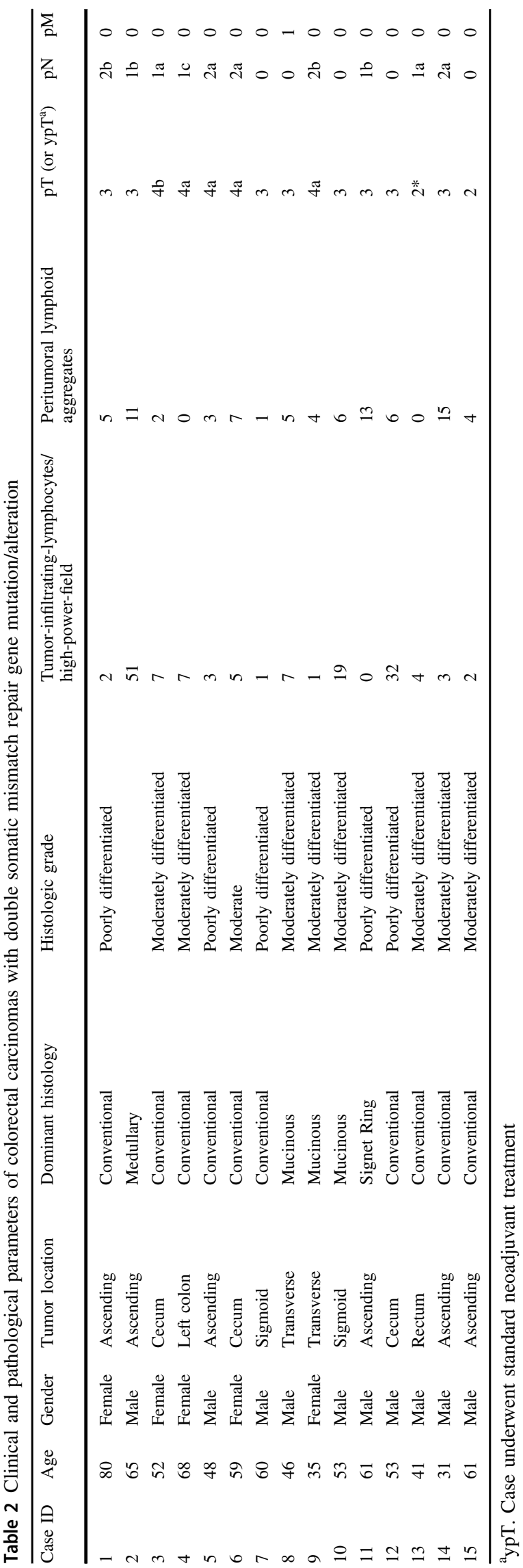

Lynch syndrome and $M L H 1$ methylated groups was 60 and $75 \%$, respectively; however, the difference reached statistical significance only between double somatic cases and MLH1 methylated cases $(p=0.006)$ and not between double somatic cases and Lynch syndrome cases $(p=0.11)$. The density of peritumoral lymphoid aggregates in double somatic mutation/alteration cases versus other types showed a similar tendency (Table 3). While Lynch syndrome and $M L H 1$ hypermethylated tumors differed in the number of peritumoral lymphoid aggregates from microsatellite stable carcinomas $(p<0.013)$, double somatic mutation/alteration cases did not $(p=0.19)$.

Cases \#2, \#6, and \#13 received treatment with a PD1/ PD-L1 checkpoint inhibitor. Case \#2 (Fig. 2) was a 65-yearold male patient whose right colon cancer was deemed to be stage IV at presentation based on radiographic evidence (CT scan, Fig. 2a), and supported by laparoscopic findings (Fig. 2b) of widespread tumor nodules in the peritoneum (largest being $9.7 \mathrm{~cm}$ in greatest dimension). Tumor sample via laparoscopic procedure was found to be mismatch repair deficient (Fig. 1 a-d). The tumor had a tumor mutation burden of $89.5 \mathrm{mut} / \mathrm{megabase}$, and carried three somatic mutations in $M S H 2$, two of which were deemed to be at least likely pathogenic (Fig. 2c). This patient enrolled in a checkpoint inhibitor trial and received anti-PD-L1 single agent treatment. Upon radiographic re-evaluation, the dominant peritoneal mass decreased in size from $9.7 \mathrm{~cm}$ to $8.2 \mathrm{~cm}$ (overall, stable disease) during the initial period. This response, however, plateaued in the $4^{\text {th }}$ month. A resection thus ensued which showed residual adenocarcinoma with features consistent with medullary carcinoma (Fig. 2d). The viable tumor showed a tumor-infiltratinglymphocyte count of 59/high-power-field. Pathological response was present, graded at $30 \%$. The response pattern was predominantly fibro-inflammatory but focally in the form of mucin pools (Fig. 2e, f). The final cancer stage was ypT3N1. The patient received conventional FOLFOX adjuvant therapy after surgery. At last follow-up, 29 months after the initial diagnosis, there was no evidence of disease.

Case \#6 was a female patient who, at age 59, underwent a right colectomy for a cecal adenocarcinoma, pT4aN2a. The tumor was gland forming, moderately differentiated, and had a tumor-infiltrating-lymphocyte count of 5/highpower-field (Fig. 1e-h). Tumor had a mutation burden of $84.6 \mathrm{mut} / \mathrm{megabase}$, and carried two $\mathrm{MSH} 2$ mutations, both deemed to be at least likely pathogenic. The patient received adjuvant chemotherapy (Capecitabine and Oxaliplatin) and remained disease free until 35 months after the initial diagnosis when she was found to have a pelvic mass, consistent with recurrent metastatic disease. This was treated with irinotecan and bevacizumab (with partial response) followed by posterior pelvic exenteration and pelvic sidewall dissection. Five months later, the patient was found to 
Table 3 Clinical and pathological characteristics of colorectal carcinomas with double somatic mismatch repair gene mutation/alteration versus other subtypes

\begin{tabular}{|c|c|c|c|c|}
\hline Tumor category & $\begin{array}{l}\text { Double somatic mutation/ } \\
\text { alteration-associated }(n=15)\end{array}$ & $\begin{array}{l}\text { Lynch syndrome-associated } \\
(n=20)\end{array}$ & $\begin{array}{l}\text { MLH1-methylated } \\
(n=20)\end{array}$ & $\begin{array}{l}\text { Microsatellite } \\
\text { stable }(n=39)\end{array}$ \\
\hline Age (mean) & 54.6 & 48.2 & 61.5 & 55.9 \\
\hline Male $(\%)$ & 66.7 & 70 & 40 & 56.4 \\
\hline \multicolumn{5}{|l|}{ Tumor side } \\
\hline Right side $(\%)$ & 73 & 75 & 95 & 36 \\
\hline Left side $(\%)$ & 27 & 25 & 5 & 63 \\
\hline \multicolumn{5}{|l|}{ Stage $(\%)$} \\
\hline $\mathrm{I} / \mathrm{II}$ & 27 & 75 & 75 & 59 \\
\hline III/IV & 73 & 25 & 25 & 41 \\
\hline \multicolumn{5}{|l|}{ Histologic patterns } \\
\hline Mucinous (\%) & 20 & 10 & 10 & 2.6 \\
\hline Medullary (\%) & 6.7 & 5 & 25 & 0 \\
\hline Poorly differentiated (\%) & 33 & 15 & 5 & 2.6 \\
\hline \multicolumn{5}{|c|}{$\begin{array}{l}\text { Tumor-infiltrating-lymphocytes / } \\
\text { high-power-field }\end{array}$} \\
\hline Median (SD) & $4(14.2)$ & $11(17.5)$ & $38(101.8)$ & $2(1.4)$ \\
\hline$\%$ cases $>=3$ & 67 & 90 & 85 & 28 \\
\hline$\%$ cases $>=10$ & 20 & 60 & 76 & 0 \\
\hline \multicolumn{5}{|c|}{ Peritumoral lymphoid aggregates } \\
\hline Median (SD) & $5(4.5)$ & $7.5(10.8)$ & $5(7.5)$ & $2(5.2)$ \\
\hline$\%$ cases $>=3$ & 73 & 65 & 70 & 33 \\
\hline$\%$ cases $>=10$ & 20 & 45 & 40 & 10 \\
\hline
\end{tabular}

have new abdominopelvic and retroperitoneal nodal recurrence as well as a liver metastasis, and initiated anti-PD1 treatment (pembrolizumab) every 3 weeks. The patient remained on this regimen until her last follow-up, 63 months after the initial diagnosis and 15 months after initiation of anti-PD1 treatment, at which point a complete response was observed with no detectable evidence of disease.

Case \#13 was a male patient, diagnosed with a locally advanced rectal cancer at age 41 . The patient underwent neoadjuvant chemo-radiation therapy followed by surgical resection. The resected tumor showed a 30\% response with residual ypT2N1 disease. The tumor was gland forming but had a minor mucinous component, with a tumor-infiltratinglymphocyte count of 4/high-power-field. Tumor had a mutation burden of 47.2 mut/megabase and carried one somatic mutation in $\mathrm{MSH} 2$ coupled with loss of heterozygosity. Fourteen months after the surgery, he was found to have recurrent tumor at the anastomotic site. This was treated with a resection, and the tumor was found to be morphologically similar to the original tumor. Ten months later, imaging showed a new tumor nodule that was $2.8 \mathrm{~cm}$ in size in the pelvis. At this point, the patient received immunotherapy with an anti-PD-L1 agent and an antiCTLA4 agent, followed by anti-PD-L1 therapy alone. A partial response was observed with the tumor decreasing in size to $1.7 \mathrm{~cm}$ at 11 months since the start of immunotherapy. The patient was then initiated on long term surveillance with imaging every 3 months. At last follow-up, 59 months after the initial diagnosis and 24 months after initiation of immunotherapy, imaging studies showed no evidence of disease.

\section{Clinico-pathological features of institutional cases versus literature cases}

The genes involved (by either double somatic mutations or mutation plus loss of heterozygosity) in both the institutional cases and the literature cases are summarized in Table 4. In total, $M L H 1$ was disproportionately more likely to be affected by loss of heterozygosity $(p=0.0012)$, occurring in $73 \%$ of the MLH1-deficient cases.

For the literature cases, detailed histological data that would allow direct comparison with our Memorial Sloan Kettering cases were not available. The average age at colorectal carcinoma diagnosis of the 68 reported cases was 56.1, similar to institutional cases (54.6). Of the 40 literature cases with available data, $57.5 \%$ were males and $82.5 \%$ were right-sided tumors, both rates were again similar to our institutional cases (50\% and $70 \%$, respectively). 

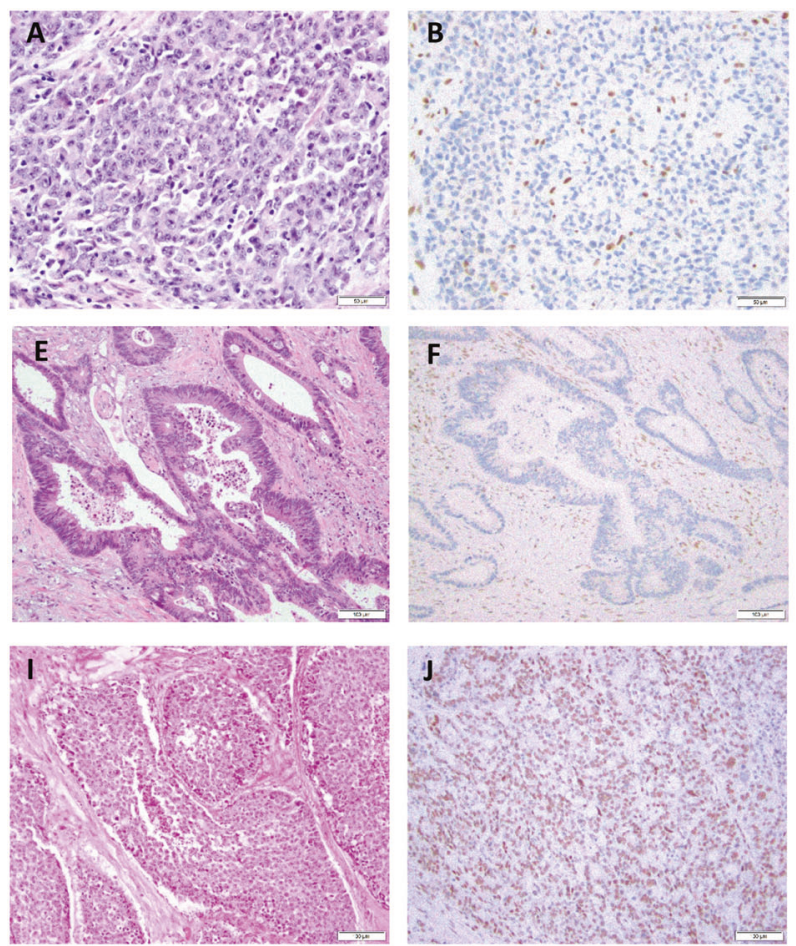

Fig. 1 Histological patterns and DNA mismatch repair protein immunohistochemistry of colorectal carcinomas with double somatic mismatch repair gene mutation/alteration. Case \#2 (a-d) exhibits a medullary pattern with tumor cells growing in sheets and nests, and containing easily discernible tumor-infiltrating-lymphocytes (a). By immunohistochemistry, this tumor shows loss of MSH2 (b) and MSH6 (c), and retained MLH1 (not illustrated) and PMS2 (d). Case \#6 (E-H)

\section{Discussion}

The clinical and biological implication of the newly recognized category of microsatellite instability-high colorectal carcinomas - the double somatic mutation/alteration tumors, is multi-fold. Most significantly, it allows affected patients to be appropriately diagnosed as not having Lynch syndrome nor "Lynch-like" syndrome. Therefore, these patients (and their family members) will no longer be automatically managed as if they had Lynch syndrome (with stringent surveillance protocols). Rather, as recommended by the current National Comprehensive Cancer Network guidelines [21], their management will be based on their personal and/or family cancer history only.

The determination that DNA mismatch repair gene somatic mutations/alteration in a colorectal carcinoma (or other tumor types) are truly deleterious and somatic (not associated with an underlying germline defect) is no trivial task, however. It requires thorough molecular, clinical, and pathological correlation [20, 22-24]. Challenging aspects include confirmation of the pathogenicity of the mutations/ alterations, confirmation of inactivation of both alleles by somatic events, and the exclusion of their association with germline alteration. The latter requires attention because it
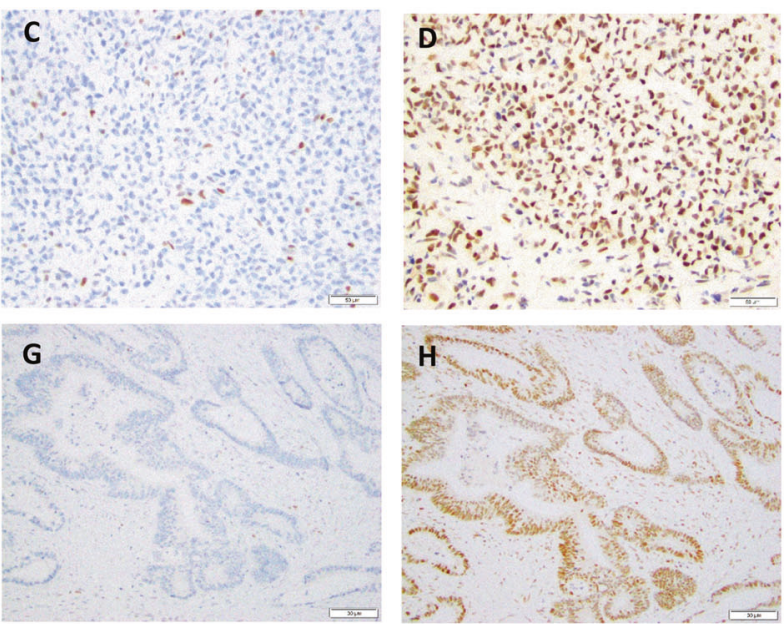

K

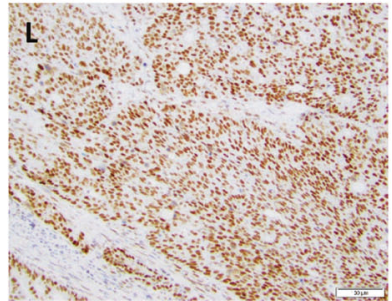

exhibits well formed glands with only very mildly elevated tumorinfiltrating-lymphocytes (e). By immunohistochemistry, this tumor shows loss of MSH2 (f) and MSH6 (g), and retained MLH1 (not illustrated) and PMS2 (h). Case \#7 (I-L) exhibits a solid growth with high grade cytology and inconspicuous tumor-infiltrating-lymphocytes (i). By immunohistochemistry, this tumor shows retained MSH2 (j), loss of MSH6 (k), and retained MLH1 (not illustrated) and PMS2 (L)

has been shown that germline mutations in either the mismatch repair genes (true Lynch syndrome) or some nonmismatch repair genes, such as MUTYH, can result in somatic inactivation of the mismatch repair genes $[18,25,26]$. Germline mutation testing is thus necessary to establish the sporadic nature. Taking such caveats into consideration, our study cases were carefully analyzed and all cases were deemed to have fulfilled the stringent inclusion criteria.

Cases thus defined, our study sought to further profile their clinical and pathological characteristics, with an emphasis on similarities or differences with other classes of mismatch repair-deficient or proficient tumors. Our results suggest that double somatic mutation/alteration colorectal carcinomas (1) tend to have an age at presentation similar to $\mathrm{MLH1}$ methylated or microsatellite stable cases and older than Lynch syndrome cases, (2) are frequently right-sided which is similar to all microsatellite instability-high tumors, (3) are more likely to present with nodal or distant metastasis than both MLH1 methylated and Lynch syndrome cases, and (4) can exhibit varied histologic characteristics with some aspects resembling microsatellite instability-high colorectal carcinomas in general, and other aspects that seem distinct such as a 

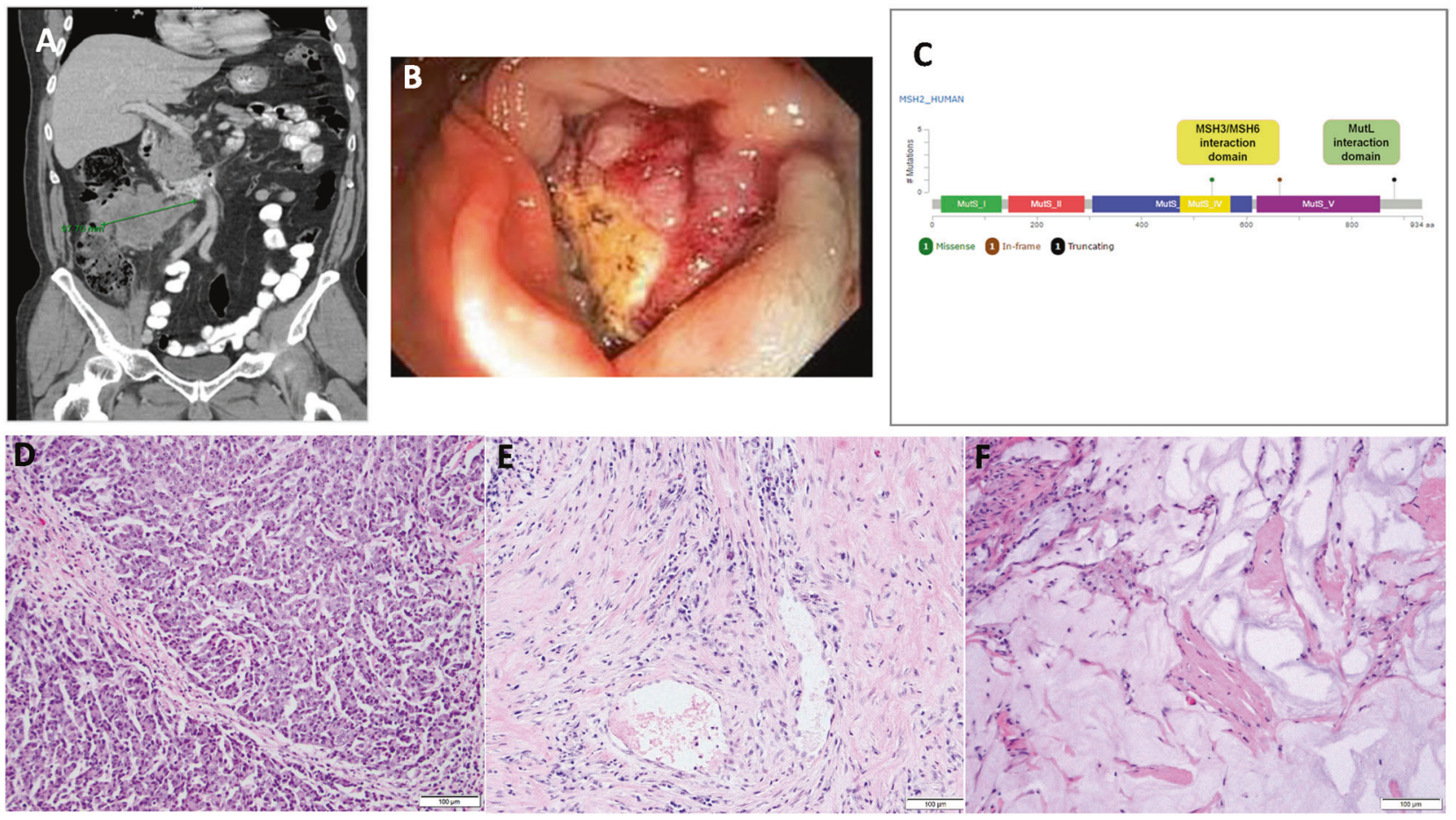

Fig. 2 Case \#2 exhibiting treatment response to immune checkpoint blockade. At presentation, CT scan shows bulky peritoneal disease (a) and endoscopy revealed a lesion in the right colon (b). By Memorial Sloan Kettering-IMPACT, the biopsy sample of the tumor revealed three somatic, likely pathogenic $M S H 2$ mutations (c). Resection of the tumor after 3 months of treatment with an anti-PD-L1 agent shows evidence of residual tumor with medullary features $(\mathbf{d}$, similar to tumor in Fig. 1a), as well as evidence of tumor regression. The latter is seen in the form of fibro-inflammatory changes in some regions (e) and acellular mucin pools in other regions (f)

Table 4 The distribution of the affected mismatch repair genes across different microsatellite instability-high colorectal carcinomas

\begin{tabular}{lllll}
\hline & $\begin{array}{l}\text { Lynch syndrome-associated } \\
(n=20, \text { institutional })\end{array}$ & $\begin{array}{l}\text { MLH1-methylated }(n=20, \\
\text { institutional })\end{array}$ & $\begin{array}{l}\text { Double somatic mutation/alteration- } \\
\text { associated }(n=15, \text { institutional })\end{array}$ & $\begin{array}{l}\text { Double somatic mutation/alteration- } \\
\text { associated }(n=68, \text { literature })\end{array}$ \\
\hline MLH1 & 9 & 20 & 6 & 43 \\
MSH2 & 4 & 0 & 8 & 22 \\
MSH6 & 2 & 0 & 2 & 4 \\
PMS2 & 5 & 0 & 0 & 1 \\
\hline
\end{tabular}

Affected genes may add up to more than the number of tumors as some tumors have more than one affected gene

proclivity to solid growth and less abundant lymphocytic infiltration.

Since the first description of double somatic mismatch repair gene mutation in colorectal carcinoma in 2013 [5], while there have been literature reports verifying this molecular mechanism as an etiology for microsatellite instability-high [16, 17, 19], systematic analysis of these tumors with regard to their clinical and pathological characteristics has been scarce. A recent study by Hemminger et al. [6] represented the first systematic evaluation of the histologic characteristics of these tumors. Hemminger et al. compared a cohort of 43 colorectal carcinomas with double somatic mutation/alteration associated mismatch repair deficiency (collected from three institutions) with 48 Lynch syndrome-associated colorectal carcinomas for the following histologic parameters: tumor-infiltrating-lymphocytes/high-power-field ( $<3$ versus $\geq 3$ ), Crohn's-like reaction (mild/moderate versus marked), necrosis, and "microsatellite instability-high histology" (defined as mucinous, signet ring, poorly differentiated, or medullary). The authors found no significant difference between the two groups and appropriately pointed out that histology is not discriminatory, and tumor sequencing for evaluation of double somatic mutations should be considered to help clarify sporadic versus hereditary causes of unexplained mismatch repair deficiency.

In line with the report by Hemminger et al. [6], we also found apparent overlapping histologic features across the three types of microsatellite instability-high colorectal carcinomas and agree that histology does not have the 
discriminatory power to distinguish the sporadic versus hereditary nature of mismatch repair deficiency. A noteworthy histologic feature observed in our analysis is that the double somatic mutation/alteration tumors had relatively low tumor-infiltrating-lymphocytes and low peritumoural lymphoid aggregates when compared to other microsatellite instability-high tumors. While a low cutoff (3 tumor-infiltrating-lymphocytes/high-power-field) did not reveal a significant difference across the three groups of microsatellite instability-high tumors (similar to the study by Hemminger et al. [6]), a higher cutoff showed that a much smaller proportion of double somatic mutation/alteration tumors had significantly elevated tumor-infiltrating-lymphocytes (Table 3). While both Lynch syndrome-associated and MLH1 methylated tumors showed more peritumoral lymphoid aggregates than microsatellite stable tumors, the double somatic mutation/alteration cases did not. Interestingly, along with this observation, we also noted an unusually high proportion of double somatic mutation/alteration colorectal carcinomas presenting with nodal or distal metastases, a phenomenon in stark contrast to the tendency for microsatellite instability-high colorectal carcinomas in general to present with node-negative disease. It is tempting to speculate that the less pronounced lymphocytic infiltration may have contributed to the more advanced stage in the double somatic mutation/alteration cases. As such, double somatic mismatch repair gene mutation/alteration may bear an adverse prognostic implication. Further studies with larger number of cases are needed to test this hypothesis. Given that microsatellite instability-high phenotype in stage IV cancers does not seem to confer a favorable outcome like it does in early stage tumors [27], another interesting question worth exploring is whether colorectal carcinomas with double somatic mismatch repair gene mutation/alteration are over-represented in stage IV microsatellite instability-high colorectal carcinomas.

From a treatment point of view, our report served to document response to PD1/PD-L1 checkpoint inhibitors in double somatic mutation/alteration associated microsatellite instability-high colorectal carcinomas. Our case \#2 offered a unique opportunity for histologic examination of primary colorectal carcinoma post anti-PD-L1 therapy as the patient received anti-PD-L1 treatment prior to the resection of primary tumor. We observed that the patterns of pathologic response to the PD-L1 inhibitor are strikingly similar to that associated with conventional chemo- or chemo-radiation therapy: fibrosis with inflammatory infiltrates and acellular mucin [28]. This suggests a potent cytotoxic effect of the immunotherapy. Our cases also illustrated that the response to this therapy may be associated with varied degrees of lymphocytic infiltration in the tumor. All three of our cases that were treated with PD-1/PD-L1 inhibition responded, yet only one had a tumor-infiltrating-lymphocyte count of 59/high-power-field while the other two had only 4 and 5 per high-power-field, respectively. This is in line with prior data that within microsatellite instability-high colorectal carcinomas, upregulation of immune checkpoint genes can occur in both tumor-infiltrating-lymphocyte -high and -low cases [29]. It is plausible that an immune cell-containing microenvironment is necessary for immune checkpoint inhibitors to exert their efficiency in colorectal carcinoma, but this environment is not purely reflected by the extent of lymphocytic infiltration. Therefore, tumor-infiltratinglymphocyte count may not serve as a sole criterion to suggest that the tumor will not respond to checkpoint blockade.

Contrast to the varied degrees of lymphocytic infiltration, the tumor mutation burden was consistently high across all colorectal carcinomas with double somatic mutation/alteration, including all three cases treated with immunotherapy. Prior studies had reported a median tumor mutation burden in microsatellite instability-high colorectal carcinoma to be 46.8/megabase (comparing to 3.6 in microsatellite stable colorectal carcinoma) [30]. Our 15 double somatic mutation/ alteration colorectal carcinomas had a median tumor mutation burden of $64.9 /$ megabase, and the mutation burden counts of the 3 patients treated with immunotherapy were $89.5,84.6$, and 47.2, respectively. While it is difficult to draw general conclusions due to the small number of cases, our results are in line with the emerging concept that tumor mutation burden can identify patients who may benefit from immune checkpoint inhibitors.

In this study, we also showed that double somatic alterations could affect any one of the four major DNA mismatch repair genes, with the most commonly affected being $M L H 1$ and $M S H 2$. This is relevant, particularly with regard to the interpretation of mismatch repair protein immunohistochemistry results. It indicates that in Lynch syndrome diagnostics, loss of MSH2, MSH6 or PMS2 protein expression by immunohistochemistry in a tumor no longer automatically implies that the case is "almost certainly" Lynch syndrome (an assumption commonly upheld in the past). Additionally, our observation of BRAF V600E mutations occurring in double somatic mutation/alteration colorectal carcinomas highlights another interpretation caveat in Lynch syndrome diagnostics: BRAF V600E in microsatellite instability-high colorectal carcinomas can occur outside the context of $M L H 1$ promoter hypermethylation as well [31]. Previous reports [32, 33] documented its occurrence in rare Lynch syndrome-associated colorectal carcinomas (genes involved included $M L H 1$ as well as MSH2 and PMS2); our study expands the spectrum of microsatellite instability-high colorectal carcinomas that could harbor $B R A F$ V600E to include those associated with double somatic mismatch repair gene mutation/alteration (genes involved may include MSH6). 
In summary, colorectal carcinomas with microsatellite instability-high secondary to double somatic mismatch repair gene mutation/alteration signify a new tumor subcategory that not only provides explanation for many previously unexplained microsatellite instability-high colorectal carcinomas (and therefore impacts Lynch syndrome work-up and management strategies), but may also bear other important clinical implications. In particular, these tumors seem to have less pronounced lymphocytic infiltration and yet a tendency towards higher TNM stage at presentation when compared to other microsatellite instability-high colorectal carcinomas, suggesting a potential prognostic connotation that is possibly mediated by lymphocytic infiltration. Intriguingly, these tumors have a consistently high tumor mutation burden, and may respond to anti-PD1/PD-L1 immunotherapy even when the lymphocytic infiltration is not striking. Additional studies with larger sample size are needed to validate these findings.

Acknowledgements This study was supported by the National Cancer Institute Cancer Center Support Grant/Core Grant P30 CA008748, and by the Romeo Milio Lynch Syndrome Foundation.

\section{Compliance with ethical standards}

Conflict of interest NS has received research funding from Roche/ Genentech, Pfizer, Merck, BMS, MedImmune/AstraZeneca, and Incyte. $\mathrm{He}$ is on the advisory board/Consulting for Roche/Genentech, Merck, BMS, MedImmune/AstraZeneca, Boehringer Ingelheim, Pfizer, Pieris, PsiOxus, Synlogic, Aduro, Kyn Therapautics, PureTech Ventures, Horizon Pharma, EMD Serono, Gritstone Oncology, Chugai, IFM therapeutics, and Imugene. LZ received honoraria from Future Technology Research LLC, Roche Diagnostics Asia Pacific and Illumina. LZ's family member has a leadership position and ownership interest of Shanghai Genome Center. JH received honorarium for a lecture from Bayer/ Loxo. DK is a consultant and equity holder of Paige.AI, and a consultant of Merck. He receives royalties from American Registry of Pathology and UpToDate. ZS reports spouse (Ophthalmology) consulting for Genentech/Roche, Novartis, Regeneron, Biomarin, Fortress Bio. JS received travel support for attending an advisory board meeting for BMS, on a topic different from the subject matter of this study. The remaining authors declatre that they have no conflict of interest.

Publisher's note: Springer Nature remains neutral with regard to jurisdictional claims in published maps and institutional affiliations.

\section{References}

1. Boland CR, Thibodeau SN, Hamilton SR, Sidransky D, Eshleman JR, Burt RW, et al. A National Cancer Institute Workshop on Microsatellite Instability for cancer detection and familial predisposition: development of international criteria for the determination of microsatellite instability in colorectal cancer. Cancer Res. 1998;58:5248-57.

2. Boland CR. Recent discoveries in the molecular genetics of Lynch syndrome. Fam Cancer. 2016;15:395-403.

3. Rosty C, Walsh MD, Lindor NM, Thibodeau SN, Mundt E, Gallinger S, et al. High prevalence of mismatch repair deficiency in prostate cancers diagnosed in mismatch repair gene mutation carriers from the colon cancer family registry. Fam Cancer. 2014;13:573-82.

4. Mensenkamp AR, Vogelaar IP, van Zelst-Stams WAG, Goossens M, Ouchene H, Hendriks-Cornelissen SJB, et al. Somatic mutations in MLH1 and MSH2 are a frequent cause of mismatch-repair deficiency in Lynch syndrome-like tumors. Gastroenterology. 2014;146:643-6.e8.

5. Sourrouille I, Coulet F, Lefevre JH, Colas C, Eyries M, Svrcek M, et al. Somatic mosaicism and double somatic hits can lead to MSI colorectal tumors. Fam Cancer. 2013;12:27-33.

6. Hemminger JA, Pearlman R, Haraldsdottir S, Knight D, Jonasson JG, Pritchard CC, et al. Histology of colorectal adenocarcinoma with double somatic mismatch repair mutations is indistinguishable from those caused by lynch syndrome. Hum Pathol. 2018;78:125-30.

7. Rigter LS, Snaebjornsson P, Rosenberg EH, Atmodimedjo PN, Aleman BM, Ten Hoeve J, et al. Double somatic mutations in mismatch repair genes are frequent in colorectal cancer after Hodgkin's lymphoma treatment. Gut. 2018;67:447-55.

8. Cheng DT, Mitchell TN, Zehir A, Shah RH, Benayed R, Syed A, et al. Memorial sloan kettering-integrated mutation profiling of actionable cancer targets (MSK-IMPACT): a hybridization capture-based next-generation sequencing clinical assay for solid tumor molecular oncology. J Mol Diagn. 2015;17:251-64.

9. Shen R, Seshan VE. FACETS: allele-specific copy number and clonal heterogeneity analysis tool for high-throughput DNA sequencing. Nucleic Acids Res. 2016;44:e131.

10. Middha S, Zhang L, Nafa K, Jayakumaran G, Wong D, Kim HR, et al. Reliable pan-cancer microsatellite instability assessment by using targeted next-generation sequencing data. JCO Precis Oncol. 2017:1-17.

11. Chakravarty D, Gao J, Phillips SM, Kundra R, Zhang H, Wang J, et al. OncoKB: A Precision Oncology Knowledge Base. JCO Precis Oncol. 2017;2017.

12. Adzhubei IA, Schmidt S, Peshkin L, Ramensky VE, Gerasimova A, Bork P, et al. A method and server for predicting damaging missense mutations. Nat Methods. 2010;7:248-9.

13. Ng PC, Henikoff S. Predicting deleterious amino acid substitutions. Genome Res. 2001;11:863-74.

14. Choi Y, Sims GE, Murphy S, Miller JR, Chan AP. Predicting the functional effect of amino acid substitutions and indels. PLoS ONE. 2012;7:e46688.

15. Cohen SA, Turner EH, Beightol MB, Jacobson A, Gooley TA, Salipante SJ, et al. Frequent PIK3CA mutations in colorectal and endometrial tumors with 2 or more somatic mutations in mismatch repair genes. Gastroenterology. 2016;151:440-7 e1.

16. Haraldsdottir S, Hampel H, Tomsic J, Frankel WL, Pearlman R, de la Chapelle A, et al. Colon and endometrial cancers with mismatch repair deficiency can arise from somatic, rather than germline, mutations. Gastroenterology. 2014;147:1308-16 e1.

17. Geurts-Giele WR, Leenen CH, Dubbink HJ, Meijssen IC, Post E, Sleddens HF, et al. Somatic aberrations of mismatch repair genes as a cause of microsatellite-unstable cancers. J Pathol. 2014;234:548-59.

18. Sourrouille I, Coulet F, Lefevre JH, Colas C, Eyries M, Svrcek M, et al. Somatic mosaicism and double somatic hits can lead to MSI colorectal tumors. Fam Cancer. 2013;12:27-33.

19. Mensenkamp AR, Vogelaar IP, van Zelst-Stams WA, Goossens M, Ouchene H, Hendriks-Cornelissen SJ, et al. Somatic mutations in MLH1 and MSH2 are a frequent cause of mismatch-repair deficiency in Lynch syndrome-like tumors. Gastroenterology. 2014;146:643-6 e8.

20. Wang T, Stadler ZK, Zhang L, Weiser MR, Basturk O, Hechtman $\mathrm{JF}$, et al. Immunohistochemical null-phenotype for mismatch repair proteins in colonic carcinoma associated with concurrent 
MLH1 hypermethylation and MSH2 somatic mutations. Fam Cancer. 2018;17:225-8.

21. NCCN Guidelines Version 3.2017. Lynch Syndrome. https://www. nccn.org/professionals/physician_gls/pdf/genetics_colon.pdf. 2017.

22. Shirts BH, Konnick EQ, Upham S, Walsh T, Ranola JMO, Jacobson $\mathrm{AL}$, et al. Using somatic mutations from tumors to classify variants in mismatch repair genes. Am J Hum Genet. 2018;103:19-29.

23. Raymond VM, Morris AM, Hafez KS, Greenson JK. MLH1 promotor hypermethylation does not rule out a diagnosis of Lynch syndrome: a case report. Fam Cancer. 2015;14:77-80.

24. Raskin L, Guo Y, Du L, Clendenning M, Rosty C, Colon Cancer Family R, et al. Targeted sequencing of established and candidate colorectal cancer genes in the Colon Cancer Family Registry Cohort. Oncotarget. 2017;8:93450-63.

25. Jansen AM, van Wezel T, van den Akker BE, Ventayol Garcia M, Ruano D, Tops CM, et al. Combined mismatch repair and POLE/ POLD1 defects explain unresolved suspected Lynch syndrome cancers. Eur J Hum Genet. 2016;24:1089-92.

26. Morak M, Heidenreich B, Keller G, Hampel H, Laner A, de la Chapelle A, et al. Biallelic MUTYH mutations can mimic Lynch syndrome. Eur J Hum Genet. 2014;22:1334-7.

27. Venderbosch S, Nagtegaal ID, Maughan TS, Smith CG, Cheadle JP, Fisher D, et al. Mismatch repair status and BRAF mutation status in metastatic colorectal cancer patients: a pooled analysis of the CAIRO, CAIRO2, COIN, and FOCUS studies. Clin Cancer Res. 2014;20:5322-30.
28. Shia J, Guillem JG, Moore HG, Tickoo SK, Qin J, Ruo L, et al. Patterns of morphologic alteration in residual rectal carcinoma following preoperative chemoradiation and their association with long-term outcome. Am J Surg Pathol. 2004;28:215-23.

29. Shia J, Schultz N, Kuk D, Vakiani E, Middha S, Segal NH, et al. Morphological characterization of colorectal cancers in The Cancer Genome Atlas reveals distinct morphology-molecular associations: clinical and biological implications. Mod Pathol. 2017;30:599-609.

30. Fabrizio DA, George TJ Jr., Dunne RF, Frampton G, Sun J, Gowen $\mathrm{K}$, et al. Beyond microsatellite testing: assessment of tumor mutational burden identifies subsets of colorectal cancer who may respond to immune checkpoint inhibition. J Gastrointest Oncol. 2018;9:610-7.

31. Pérez-Carbonell L, Alenda C, Payá A, Castillejo A, Barberá VM, Guillén C, et al. Methylation analysis of MLH1 improves the selection of patients for genetic testing in Lynch syndrome. J Mol Diagn. 2010;12:498-504.

32. Senter L, Clendenning M, Sotamaa K, Hampel H, Green J, Potter JD, et al. The clinical phenotype of Lynch syndrome due to germline PMS2 mutations. Gastroenterology. 2008;135:419-28.

33. Parsons MT, Buchanan DD, Thompson B, Young JP, Spurdle AB. Correlation of tumour BRAF mutations and MLH1 methylation with germline mismatch repair (MMR) gene mutation status: a literature review assessing utility of tumour features for MMR variant classification. J Med Genet. 2012;49:151-7. 